\title{
Review Article \\ Crosstalk between Tryptophan Metabolism and Cardiovascular Disease, Mechanisms, and Therapeutic Implications
}

\author{
Gang Liu, ${ }^{1}$ Shuai Chen, ${ }^{1}$ Jin Zhong, ${ }^{2,3}$ Kunling Teng, ${ }^{2}$ and Yulong Yin ${ }^{1,4,5}$ \\ ${ }^{1}$ Key Laboratory of Agro-Ecological Processes in Subtropical Region, Institute of Subtropical Agriculture, \\ Chinese Academy of Sciences, National Engineering Laboratory for Pollution Control and Waste Utilization in Livestock and \\ Poultry Production, Hunan Provincial Engineering Research Center of Healthy Livestock, Scientific Observing and \\ Experimental Station of Animal Nutrition and Feed Science in South-Central, Ministry of Agriculture, \\ Hunan Co-Innovation Center of Animal Production Safety, Hunan 410125, China \\ ${ }^{2}$ State Key Laboratory of Microbial Resources, Institute of Microbiology, Chinese Academy of Sciences, Beijing 100101, China \\ ${ }^{3}$ University of Chinese Academy of Sciences, Beijing 100049, China \\ ${ }^{4}$ Laboratory of Animal Nutrition and Human Health, School of Biology, Hunan Normal University, Changsha, Hunan, China \\ ${ }^{5}$ College of Animal Science, South China Agricultural University, Guangzhou 510642, China
}

Correspondence should be addressed to Gang Liu; gangle.liu@gmail.com, Jin Zhong; zhongj@im.ac.cn, and Yulong Yin; yinyulong@isa.ac.cn

Received 19 January 2017; Accepted 16 February 2017; Published 9 March 2017

Academic Editor: Alexander N. Orekhov

Copyright (C) 2017 Gang Liu et al. This is an open access article distributed under the Creative Commons Attribution License, which permits unrestricted use, distribution, and reproduction in any medium, provided the original work is properly cited.

The cardiovascular diseases (CVD) associated with the highest rates of morbidity are coronary heart disease and stroke, and the primary etiological factor leading to these conditions is atherosclerosis. This long-lasting inflammatory disease, characterized by how it affects the artery wall, results from maladaptive immune responses linked to the vessel wall. Tryptophan (Trp) is oxidized in a constitutive manner by tryptophan 2,3-dioxygenase in liver cells, and for alternative cell types, it is catalyzed in the presence of a differently inducible indoleamine 2,3-dioxygenase (IDO1) in the context of a specific pathophysiological environment. Resultantly, this leads to a rise in the production of kynurenine (Kyn) metabolites. Inflammation in the preliminary stages of atherosclerosis has a significant impact on IDO1, and IDO1 and the IDO1-associated pathway constitute critical mediating agents associated with the immunoinflammatory responses that characterize advanced atherosclerosis. The purpose of this review is to survey the recent literature addressing the kynurenine pathway of tryptophan degradation in CVD, and the author will direct attention to the function performed by IDO1-mediated tryptophan metabolism.

\section{Introduction}

Tryptophan (Trp), an essential amino acid, constitutes a central component in human and animal protein synthesis, and it serves as the sole source of substrates that facilitate the generation of a range of crucial molecules. Trp precedes and indicates the synthesis of proteins, nicotinamide adenine dinucleotide (NAD), nicotinic acid, and serotonin (namely, the neurotransmitter) $[1,2]$. For mammalian species, the kynurenine (Kyn) pathway is Trp's central catabolic route, featured in $95 \%$ of peripheral Trp metabolism in mammals; furthermore, it results in NAD's biosynthesis, as NAD functions as a crucial cofactor [3].
The highest rates of global morbidity are associated with cardiovascular disease (CVD), and atherosclerosis is the primary etiological factor leading to various manifestations of CVD, including coronary heart disease and stroke [4]. One of the critical factors in CVD pathogenesis is the immune response, and a clinical solution remains to be identified $[5,6]$. Atherosclerosis occurs due to the manner in which low-density lipoprotein (LDL) accumulates and is retained in the arterial wall, and this leads to maladaptive responses from $\mathrm{T}$ cells and macrophages [7]. Scholars in recent years have directed significant energy towards the examination of the Kyn pathway and the role it plays in CVD pathogenesis, and because several hypotheses have suggested that various 
factors, including oxidative stress, immune activation, and inflammation, are central to the pathogenesis of atherosclerosis and CVD, a critical area of future investigation is to examine to potential part played by the Kyn pathway in CVD regarding these factors.

\section{Tryptophan Metabolism and the Kynurenine Pathway}

Trp hydroxylase facilitates the biotransformation of approximately 5\% Trp via metabolism to 5-hydroxy Trp, and this generates serotonin by decarboxylase (an amino acid). Lastly, through $N$-acetyltransferase, serotonin is metabolized to melatonin. Via the Kyn pathway, the degradation of the other $95 \%$ of $\operatorname{Trp}$ is converted to kynurenine, and the regulation of this primarily occurs with a pair of ratelimiting enzymes, tryptophan 2,3-dioxygenase (TDO) and indoleamine 2,3-dioxygenase (IDO1). Each of these enzymes incorporates one noncovalently bound iron-protoporphyrin IX to every monomer, and TDO and IDO1 are members of the oxidoreductase family. Specifically, the enzymes are associated with the family of oxidoreductases that act on single donors with $\mathrm{O}_{2}$ as the oxidant and the inclusion of two oxygen atoms into the substrate (oxygenases) $[8,9]$. The expression of IDO1 occurs at basal levels as a consequence of antigen-presenting cells, including macrophages and dendritic cells, and this procedure is driven to a significant extent by IFN- $\gamma$, the proinflammatory cytokine, and type I interferons, tumor necrosis factor, and lipopolysaccharide (LPS) (the latter three to a less significant degree) [10]. Considerable scholarly attention has been directed towards the immunoregulatory function played by Trp metabolism in the immune system, and most studies have centered on the role of IDO1; this rate-limiting enzyme governs the ratelimiting step of Trp catabolism. Kynureninase, after it has been synthesized by IDO1, uses Kyn to generate anthranilic acid (AA) [11]. Additional steps in the Kyn pathway relate to the degradation of kynurenine to the sequential production of 3-hydroxybutyrate kynurenine and 3-hydroxybutyrate anthranilic acid (3-HAA) or xanthurenic acid in the presence of kynurenine-3-monooxygenase (KMO) and kynureninase or kynurenine aminotransferase, respectively. 3-HAA is further metabolized to quinolinic acid (QA), the excitotoxin, which is a potent convulsant and excitant [12]. Furthermore, studies have demonstrated that kynurenine aminotransferase metabolizes Kyn to generate kynurenic acid (KYNA) [13]. Due to its N-methyl-D-aspartate (NMDA) receptor antagonist characteristics, KYNA is a neuroprotective compound [12]. The manner in which KMO is expressed and acts is improved by IFN- $\gamma$ in the context of human macrophages and microglia cells [14], and an increase in KMO expression is linked to significant levels of TNF- $\alpha$ and IL- 6 in the brains of rats after a systemic inflammatory challenge [15]. Figure 1 provides a schematic illustration of the ways in which the critical enzymes and substrates linked to the Trp metabolic pathway are associated with one another [9], and it also demonstrates the primary immune-related active substances, including kynurenine, quinolinic acid, 5-hydroxytryptamine (5-HT), and melatonin.

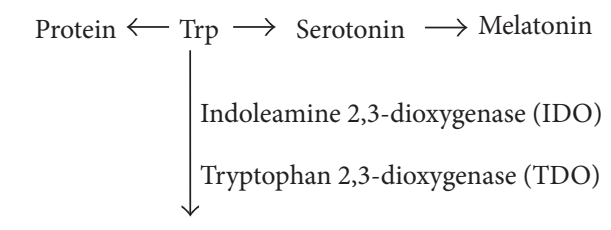

N-Formyl kynurenine

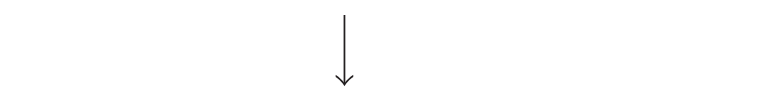

Kynureninic acid $\longleftarrow$ Kynurenine $\longrightarrow$ Anthranilic acid<smiles>C1CCCCC1</smiles>

3-OH kynurenine $\longrightarrow$ Xanthurenic acid<smiles>C1CCCCC1</smiles>

3-Hydroxybutyrate anthranilic acid

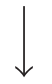

Quinolinic acid

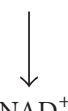

FIGURE 1: Schematic illustration of Trp catabolism along the mammalian Kyn pathway.

Preliminary research in this area mainly attributed the Kyn pathway with a central function in the generation of nicotinic acid or vitamin B3 [16]. Nevertheless, after the observation that modifications of Trp metabolism are present in numerous central nervous system conditions, attention moved towards the produced enzymes and metabolites, subsequently denoted as kynurenines. One of the critical findings was that QA operates as a potent convulsant and excitant [12] and, as such, resulted in convulsive responses when inserted into mouse brain ventricles. Furthermore, researchers found that QA functions as a selective NMDA receptor agonist [17]. AS, Trp, Kyn, AA, 3-hydroxybutyrate kynurenine, and xanthurenic acid readily cross the blood-brain barrier $[18,19]$. The impacts that systemic Trp has on the brain Kyn pathway is partly facilitated by its peripheral conversion to Kyn and 3-OHkyn. An additional driver ensures entry of these metabolites into the brain. Kynurenic acid, 3-HAA, and QA, primarily as a consequence of the polar nature and the seeming absence of effective transition procedures, are not the same as a range of different kynurenine pathway metabolites because they cannot effectively cross the blood-brain barrier [18]. Therefore, their formation occurs in a local manner inside the brain.

\section{Kynurenine Pathway and Immune Responses}

Research has identified that a key function of the Kyn pathway relates to the pathological regulation of the innate and adaptive immune system [3]. In a prospective multicenter study 
involving a 986-person sample group, comprised entirely of individuals in the young adult age range, investigators noted that the activity of IDO1 is significantly associated with carotid artery intima-media thickness (IMT) in females. Specifically, IDO1 activity displayed a significant association with a range of atherosclerosis risk factors for the female population, including age, LDL cholesterol (LDL-C), and BMI. Moreover, IFN- $\gamma$ was identified as the primary IDO1 inducer in vitro and in vivo, and the presence of IFN- $\gamma$ facilitated an increase in intracellular IDO1 transcription [20, 21]. Another study identified alternative inflammatory factors as less prominent inducers of IDO1, including IFN- $\alpha$, IFN- $\beta$, LPS, and cytotoxic T lymphocyte-associated antigen-4 [22].

Contemporary research findings have contributed to a body of knowledge in which a minimum of three mechanisms that facilitate the initiation of immunological suppression are understood. It is notable that all of the identified immunosuppressive impacts are aligned with IDO1 activation and its downstream effects on specific groups of T cells. Initially, active IDO1 facilitates the depletion of Trp in local tissue microenvironments and, in turn, it drives the promotion of metabolite generation associated with the Kyn pathway. After IDO1 induction, which results in the inhibition of the propagation of reactive $\mathrm{T}$ lymphocytes, Trp levels are depleted, which increases the degree to which $\mathrm{T}$ lymphocytes are susceptible to cell death [23]. In vitro Trp depletion leads to cell cycle impedance of activated T cells, and this similarly increases the likelihood of cell death [24].

Second, the subsequent rise in kynurenine metabolites, including Kyn, QA, and 3-HAA, impedes propagation and, following this, facilitates the initiation of selective cell death regarding $\mathrm{T}$ helper 1 (TH1) lymphocytes, which respond to antigen-presenting cells [25]. Research findings have demonstrated that kynurenine results in negative impacts regarding several phenomena, including how immune responses are regulated, the inhibition of $\mathrm{T}$ cell and NK cell propagation, and the regulation of immunogenic dendritic cells [26]. In the context of inflammation conditions, Trp degrades quickly to QA, and QA and 3-hydroxybutyrate kynurenine have the potential to induce the selective cell death in vitro of TH1 and not TH2 cells. Therefore, by suppressing and removing T lymphocytes, Trp metabolism has the potential to impact immunity [27].

Third, it is important to recognize that an increase in the frequency of regulatory $\mathrm{T}$ cells positive for forkhead box $\mathrm{P} 3$ (FOXP3+) via TGF $\beta$ induction occurs when two conditions, namely, the presence of kynurenine metabolites and $\operatorname{Trp}$ depletion, are met, and the simultaneous presence of these conditions also heightens the effect on naïve T cells [27]. This effect significantly advances immune tolerance and a negatively formulated feedback loop, thereby driving immune response regulation [28].

\section{Kynurenine Pathway and Cardiovascular Disease}

The overexpression of IDO1 accompanied by increased Trp catabolism has been shown to stem from chronic systemic low-grade inflammation (CSLGI), which is a predictive factor for the results of CVD. The enhanced degradation of Trp was linked to inflammation in [29] by an observation of the increased plasma Kyn to Trp ratio (Kyn/Trp) (KTR). Here, a sample group of mature participants from Finland demonstrated that the aforementioned ratio is positively associated with BMI, LDL, triglycerides, and waist circumference while it is negatively associated with high-density lipoprotein (HDL) [30]. Studies were conducted on an expansive cohort taking a broad sample of all demographic groups, and it was noted that IDO1 activity (via the KTR) was positively associated with the preliminary stages of atherosclerosis and increased carotid artery IMT for males and females; this finding indicates that IDO1 constitutes a viable indicator of atherosclerosis [31]. Increased IDO1 expression was identified in the macrophage-loaded core of atherosclerotic plaques in human participants [32], and another study demonstrated that low Trp plasma concentration and a high KTR are characteristic of individuals suffering from coronary heart disease [33]. Moreover, a high KTR is a sensitive indicator of severe coronary events for individuals displaying no history of coronary artery disease [34]. Therefore, KTR can be used to forecast critical coronary events and is also useful in determining all-cause mortality for individuals suffering from coronary artery disease [35]. A relationship was observed between KTR and IMT for individuals suffering from hemodialysis while being classified as high risk for CVD [36], and it was reported that increased Trp degradation is associated with neopterin plasma concentrations [35]. This finding constitutes a biomarker of cell-mediated immune activation and is connected to atherosclerotic CVD [37]. Epidemiological research indicates that the Kyn pathway's activity, as manifested in the plasma Kyn/Trp, is associated with the strokeinduced inflammatory response, the degree of which strokes is severe, and chronic clinical results [38]. Kyn, which has been shown to precede and indicate KYNA, was identified as considerably reducing neuronal damage and infarct volume, and this was determined in a study involving the preischemic intraperitoneal administration in various rat models of brain ischemia-hypoxia [39]. Furthermore, 3-hydroxykynurenine, as is the case with the KTR, has been linked to the appearance of CVD in individuals suffering from chronic renal disease, and this was verified in an independent manner [40].

In preliminary investigations, 3-HAA was frequently denoted as a Trp metabolite resulting in antioxidant and antiinflammatory impacts. Research has shown that 3-HAA in mitochondrial mechanisms impedes oxygen uptake by mitochondrial respiration with NAD-dependent substrates, the uncoupling of the respiratory chain, and oxidative phosphorylation [41]. In addition, various studies have examined the ways in which 3-HAA results in cell death, by associating it with the apoptosis induction in monocyte/macrophage cell lines [42], identifying a link between 3-HAA and apoptosis in activated $\mathrm{T}$ cells [43], and demonstrating that 3-HAA facilitates the inhibition of nuclear factor- $\kappa \mathrm{B}$ activation [44]. Experimental findings indicate that 3-HAA has a significant function regarding atheroprotection in that it facilitates the regulation of lipoprotein metabolism. $\mathrm{LDLr}^{-/-}$and $\mathrm{IDO}^{-/-}$ double knockout mice displayed a considerable increase 
in serum lipids, especially triglycerides [45]. Furthermore, the administration of 3-HAA to $\mathrm{LDL}$ receptor knockout $\left(\mathrm{LDLr}^{-/-}\right)$mice facilitated a significant decrease in overall plasma cholesterol and triglyceride levels, and the former effect was attributed to the lower chylomicron/VLDL ratio. In addition, 3-HAA led to a significant increase in HDL-C [46].

\section{Therapeutic Implications and Concluding Remarks}

CSLGI linked to conventional CVD risk factors leads to an increase in Trp degradation. Therefore, one of the critical objectives in developing appropriate therapies for the symptoms of CVD patients is to normalize Trp metabolism. Practitioners should be aware that a high KTR may indicate a natural immune reaction, meant to combat inflammation and, furthermore, that the Kyn pathway can modulate vascular inflammation and atherosclerosis in a direct or indirect manner. Considering that IDO1 inhibition facilitates a reversal in septic shock associated-hypotension and, moreover, diminishes the likelihood of fatality, Kyn constitutes a fruitful area of investigation regarding the creation of therapies for hypertension.

More research is needed to gain comprehensive insight into the function of the Kyn pathway in the modulation of cardiovascular risk factors, atherosclerosis, and vascular inflammation. Furthermore, the nature of the Kyn pathway's related and initiated molecular mechanisms of action require more in-depth research. Specifically, future studies should focus on an investigation of the degree to which these parameters constitute the potential foundation of accurate and effective biologically informed therapies that can be implemented to heighten the likelihood of patient recovery from CVD.

\section{Competing Interests}

The authors declare that there is no conflict of interests regarding the publication of this article.

\section{Acknowledgments}

This research was supported by International Partnership Program of Chinese Academy of Sciences (161343KYSB20160008), National Key Research and Development Program of China (2016YFD0500504), National Natural Science Foundation of China (nos. 31570114, 31330075, 31110103909, 31572416, 31402092, and 31372326), Ministry of Agricultural of the People's Republic of China (2015-Z64, 2016-X47), Special Fund for Agro-scientific Research in the Public Interest (201503134), and Hunan Provincial Science and Technology Department (2016NK2101, 2016WK2008, and 2016TP2005).

\section{References}

[1] G. F. Oxenkrug, "Genetic and hormonal regulation of tryptophan-kynurenine metabolism: implications for vascular cognitive impairment, major depressive disorder, and aging," Annals of the New York Academy of Sciences, vol. 1122, pp. 35-49, 2007.
[2] D. A. Chistiakov, A. N. Orekhov, and Y. V. Bobryshevy, "Cardiac extracellular vesicles in normal and infarcted heart," International Journal of Molecular Sciences, vol. 17, no. 1, article 63, 2016.

[3] L. Vécsei, L. Szalárdy, F. Fülöp, and J. Toldi, "Kynurenines in the CNS: recent advances and new questions," Nature Reviews Drug Discovery, vol. 12, no. 1, pp. 64-82, 2013.

[4] M. Nichols, N. Townsend, P. Scarborough, and M. Rayner, "Cardiovascular disease in Europe 2014: epidemiological update," European Heart Journal, vol. 35, no. 42, pp. 2950-2959, 2014.

[5] J. E. Cole, N. Astola, A. P. Cribbs et al., "Indoleamine 2,3-dioxygenase- 1 is protective in atherosclerosis and its metabolites provide new opportunities for drug development," Proceedings of the National Academy of Sciences of the United States of America, vol. 112, no. 42, pp. 13033-13038, 2015.

[6] A. N. Orekhov, "Mechanisms of atherogenesis and development of anti-atherosclerotic therapy," Current Pharmaceutical Design, vol. 21, no. 9, pp. 1103-1106, 2015.

[7] I. Tabas, K. J. Williams, and J. Borén, "Subendothelial lipoprotein retention as the initiating process in atherosclerosis: update and therapeutic implications," Circulation, vol. 116, no. 16, pp. 1832-1844, 2007.

[8] N. J. C. King and S. R. Thomas, "Molecules in focus: indoleamine 2,3-dioxygenase," International Journal of Biochemistry and Cell Biology, vol. 39, no. 12, pp. 2167-2172, 2007.

[9] O. Takikawa, "Biochemical and medical aspects of the indoleamine 2,3-dioxygenase- initiated L-tryptophan metabolism," Biochemical and Biophysical Research Communications, vol. 338, no. 1, pp. 12-19, 2005.

[10] G. Werner-Felmayer, E. R. Werner, D. Fuchs, A. Hausen, G. Reibnegger, and H. Wachter, "Neopterin formation and tryptophan degradation by a human myelomonocytic cell line (THP1) upon cytokine treatment," Cancer Research, vol. 50, no. 10, pp. 2863-2867, 1990.

[11] H. A. Walsh and N. P. Botting, "Purification and biochemical characterization of some of the properties of recombinant human kynureninase," European Journal of Biochemistry, vol. 269, no. 8, pp. 2069-2074, 2002.

[12] R. Schwarcz and R. Pellicciari, "Manipulation of brain kynurenines: glial targets, neuronal effects, and clinical opportunities," Journal of Pharmacology and Experimental Therapeutics, vol. 303, no. 1, pp. 1-10, 2002.

[13] T. W. Stone and L. G. Darlington, "Endogenous kynurenines as targets for drug discovery and development," Nature Reviews Drug Discovery, vol. 1, no. 8, pp. 609-620, 2002.

[14] A. Chiarugi, M. Calvani, E. Meli, E. Traggiai, and F. Moroni, "Synthesis and release of neurotoxic kynurenine metabolites by human monocyte-derived macrophages," Journal of Neuroimmunology, vol. 120, no. 1-2, pp. 190-198, 2001.

[15] T. J. Connor, N. Starr, J. B. O’Sullivan, and A. Harkin, "Induction of indolamine 2,3-dioxygenase and kynurenine 3-monooxygenase in rat brain following a systemic inflammatory challenge: a role for IFN- $\gamma$ ?" Neuroscience Letters, vol. 441, no. 1, pp. 29-34, 2008.

[16] R. Schwarcz, "The kynurenine pathway of tryptophan degradation as a drug target," Current Opinion in Pharmacology, vol. 4, no. 1, pp. 12-17, 2004.

[17] T. W. Stone and M. N. Perkins, "Quinolinic acid: a potent endogenous excitant at amino acid receptors in CNS," European Journal of Pharmacology, vol. 72, no. 4, pp. 411-412, 1981. 
[18] R. Schwarcz, J. P. Bruno, P. J. Muchowski, and H.-Q. Wu, "Kynurenines in the mammalian brain: when physiology meets pathology," Nature Reviews Neuroscience, vol. 13, no. 7, pp. 465477, 2012.

[19] L. Z. Agudelo, T. Femenia, F. Orhan et al., "Skeletal muscle PGC-1 alpha 1 modulates kynurenine metabolism and mediates resilience to stress-induced depression," Cell, vol. 160, no. 1-2, pp. 351-351, 2015.

[20] Y.-I. Jeong, S. W. Kim, I. D. Jung et al., "Curcumin suppresses the induction of indoleamine 2,3-dioxygenase by blocking the Janus-activated kinase-protein kinase C $\delta$-STAT1 signaling pathway in interferon- $\gamma$-stimulated murine dendritic cells," Journal of Biological Chemistry, vol. 284, no. 6, pp. 3700-3708, 2009.

[21] M. C. Cuffy, A. M. Silverio, L. Qin et al., "Induction of indoleamine 2,3-dioxygenase in vascular smooth muscle cells by interferon $\gamma$ contributes to medial immunoprivilege," Journal of Immunology, vol. 179, no. 8, pp. 5246-5254, 2007.

[22] A. L. Mellor and D. H. Munn, "IDO expression by dendritic cells: tolerance and tryptophan catabolism," Nature Reviews Immunology, vol. 4, no. 10, pp. 762-774, 2004.

[23] D. H. Munn, E. Shafizadeh, J. T. Attwood, I. Bondarev, A. Pashine, and A. L. Mellor, "Inhibition of T cell proliferation by macrophage tryptophan catabolism," Journal of Experimental Medicine, vol. 189, no. 9, pp. 1363-1372, 1999.

[24] G. K. Lee, H. J. Park, M. MacLeod, P. Chandler, D. H. Munn, and A. L. Mellor, "Tryptophan deprivation sensitizes activated T cells to apoptosis prior to cell division," Immunology, vol. 107, no. 4, pp. 452-460, 2002.

[25] P. Terness, T. M. Bauer, L. Röse et al., "Inhibition of allogeneic T cell proliferation by indoleamine 2,3-dioxygenase-expressing dendritic cells: mediation of suppression by tryptophan metabolites," Journal of Experimental Medicine, vol. 196, no. 4, pp. 447-457, 2002.

[26] J. D. Mezrich, J. H. Fechner, X. Zhang, B. P. Johnson, W. J. Burlingham, and C. A. Bradfield, "An interaction between kynurenine and the aryl hydrocarbon receptor can generate regulatory T cells," Journal of Immunology, vol. 185, no. 6, pp. 31903198, 2010.

[27] F. Fallarino, U. Grohmann, S. You et al., "The combined effects of tryptophan starvation and tryptophan catabolites downregulate $\mathrm{T}$ cell receptor $\zeta$-chain and induce a regulatory phenotype in naive T cells," Journal of Immunology, vol. 176, no. 11, pp. 6752-6761, 2006.

[28] A. L. Colín-González, P. D. Maldonado, and A. Santamaría, "3-Hydroxykynurenine: an intriguing molecule exerting dual actions in the Central Nervous System," NeuroToxicology, vol. 34, no. 1, pp. 189-204, 2013.

[29] H. Mangge, K. L. Summers, A. Meinitzer et al., "Obesity-related dysregulation of the Tryptophan-Kynurenine metabolism: role of age and parameters of the metabolic syndrome," Obesity, vol. 22, no. 1, pp. 195-201, 2014.

[30] M. Pertovaara, A. Raitala, M. Juonala et al., "Indoleamine 2,3dioxygenase enzyme activity correlates with risk factors for atherosclerosis: the cardiovascular risk in young finns study," Clinical and Experimental Immunology, vol. 148, no. 1, pp. 106111, 2007.

[31] P. Niinisalo, A. Raitala, M. Pertovaara et al., "Indoleamine 2,3dioxygenase activity associates with cardiovascular risk factors: the health 2000 study," Scandinavian Journal of Clinical and Laboratory Investigation, vol. 68, no. 8, pp. 767-770, 2008.
[32] P. Niinisalo, N. Oksala, M. Levula et al., "Activation of indoleamine 2,3-dioxygenase-induced tryptophan degradation in advanced atherosclerotic plaques: tampere Vascular Study," Annals of Medicine, vol. 42, no. 1, pp. 55-63, 2010.

[33] B. Wirleitner, V. Rudzite, G. Neurauter et al., "Immune activation and degradation of tryptophan in coronary heart disease," European Journal of Clinical Investigation, vol. 33, no. 7, pp. 550554, 2003.

[34] G. Sulo, S. E. Vollset, O. Nygard et al., "Neopterin and kynurenine-tryptophan ratio as predictors of coronary events in older adults, the Hordaland Health Study," International Journal of Cardiology, vol. 168, no. 2, pp. 1435-1440, 2013.

[35] E. R. Pedersen, Ø. Midttun, P. M. Ueland et al., "Systemic markers of interferon- $\gamma$-mediated immune activation and longterm prognosis in patients with stable coronary artery disease," Arteriosclerosis, Thrombosis, and Vascular Biology, vol. 31, no. 3, pp. 698-704, 2011

[36] A. Kato, Y. Suzuki, T. Suda et al., "Relationship between an increased serum kynurenine/tryptophan ratio and atherosclerotic parameters in hemodialysis patients," Hemodialysis International, vol. 14, no. 4, pp. 418-424, 2010.

[37] K. K. Ray, D. A. Morrow, M. S. Sabatine et al., "Long-term prognostic value of neopterin: a novel marker of monocyte activation in patients with acute coronary syndrome," Circulation, vol. 115, no. 24, pp. 3071-3078, 2007.

[38] R. Brouns, R. Verkerk, T. Aerts et al., "The role of tryptophan catabolism along the kynurenine pathway in acute ischemic stroke," Neurochemical Research, vol. 35, no. 9, pp. 1315-1322, 2010.

[39] K. Sas, H. Robotka, É. Rózsa et al., "Kynurenine diminishes the ischemia-induced histological and electrophysiological deficits in the rat hippocampus," Neurobiology of Disease, vol. 32, no. 2, pp. 302-308, 2008.

[40] D. Pawlak, T. Domaniewski, M. Mysliwiec, and K. Pawlak, "Inflammation and the prevalence of cardiovascular disease in patients with end-stage renal disease," Atherosclerosis Supplements, vol. 11, no. 2, pp. 41-41, 2010.

[41] T. Ishii, H. Iwahashi, R. Sugata, and R. Kido, "Formation of hydroxanthommatin-derived radical in the oxidation of 3-hydroxykynurenine," Archives of Biochemistry and Biophysics, vol. 294, no. 2, pp. 616-622, 1992.

[42] T. Morita, K. Saito, M. Takemura et al., “3-Hydroxyanthranilic acid, an L-tryptophan metabolite, induces apoptosis in monocyte-derived cells stimulated by interferon- $\gamma$," Annals of Clinical Biochemistry, vol. 38, no. 3, pp. 242-251, 2001.

[43] S.-M. Lee, Y.-S. Lee, J.-H. Choi et al., "Tryptophan metabolite 3-hydroxyanthranilic acid selectively induces activated $\mathrm{T}$ cell death via intracellular GSH depletion," Immunology Letters, vol. 132, no. 1-2, pp. 53-60, 2010.

[44] T. Hayashi, J.-H. Mo, X. Gong et al., “3-Hydroxyanthranilic acid inhibits PDK1 activation and suppresses experimental asthma by inducing T cell apoptosis," Proceedings of the National Academy of Sciences of the United States of America, vol. 104, no. 47, pp. 18619-18624, 2007.

[45] M. Y. Chang, C. Smith, J. B. DuHadaway et al., "Cardiac and gastrointestinal liabilities caused by deficiency in the immune modulatory enzyme indoleamine 2,3-dioxygenase," Cancer Biology and Therapy, vol. 12, no. 12, pp. 1050-1058, 2011.

[46] L. Zhang, O. Ovchinnikova, A. Jönsson et al., "The tryptophan metabolite 3-hydroxyanthranilic acid lowers plasma lipids and decreases atherosclerosis in hypercholesterolaemic mice," European Heart Journal, vol. 33, no. 16, pp. 2025-2034, 2012. 


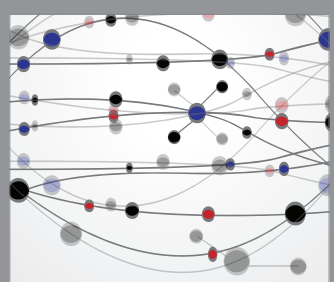

The Scientific World Journal
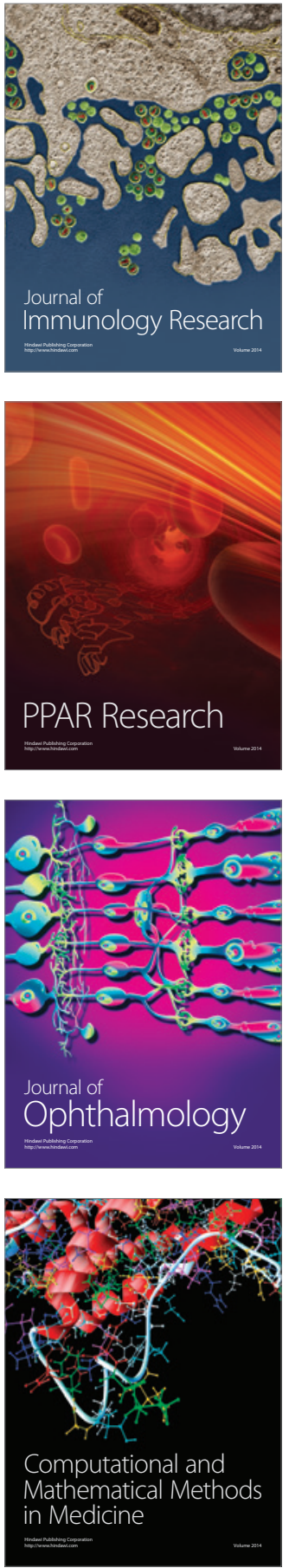

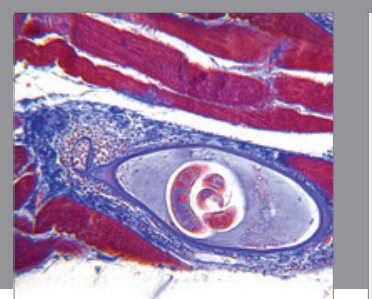

Gastroenterology Research and Practice
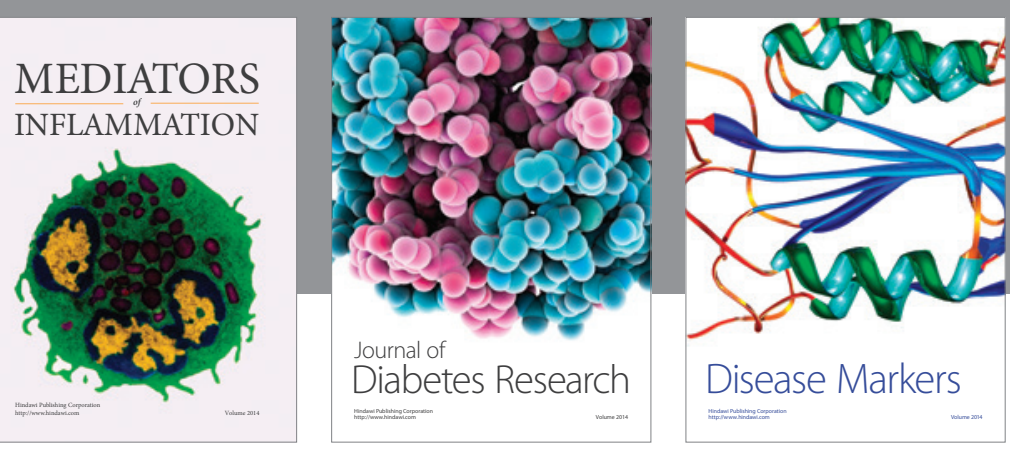

Disease Markers

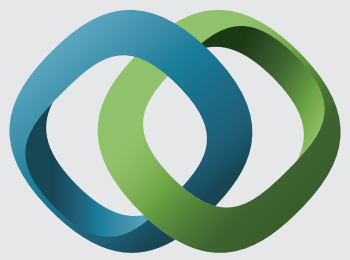

\section{Hindawi}

Submit your manuscripts at

https://www.hindawi.com
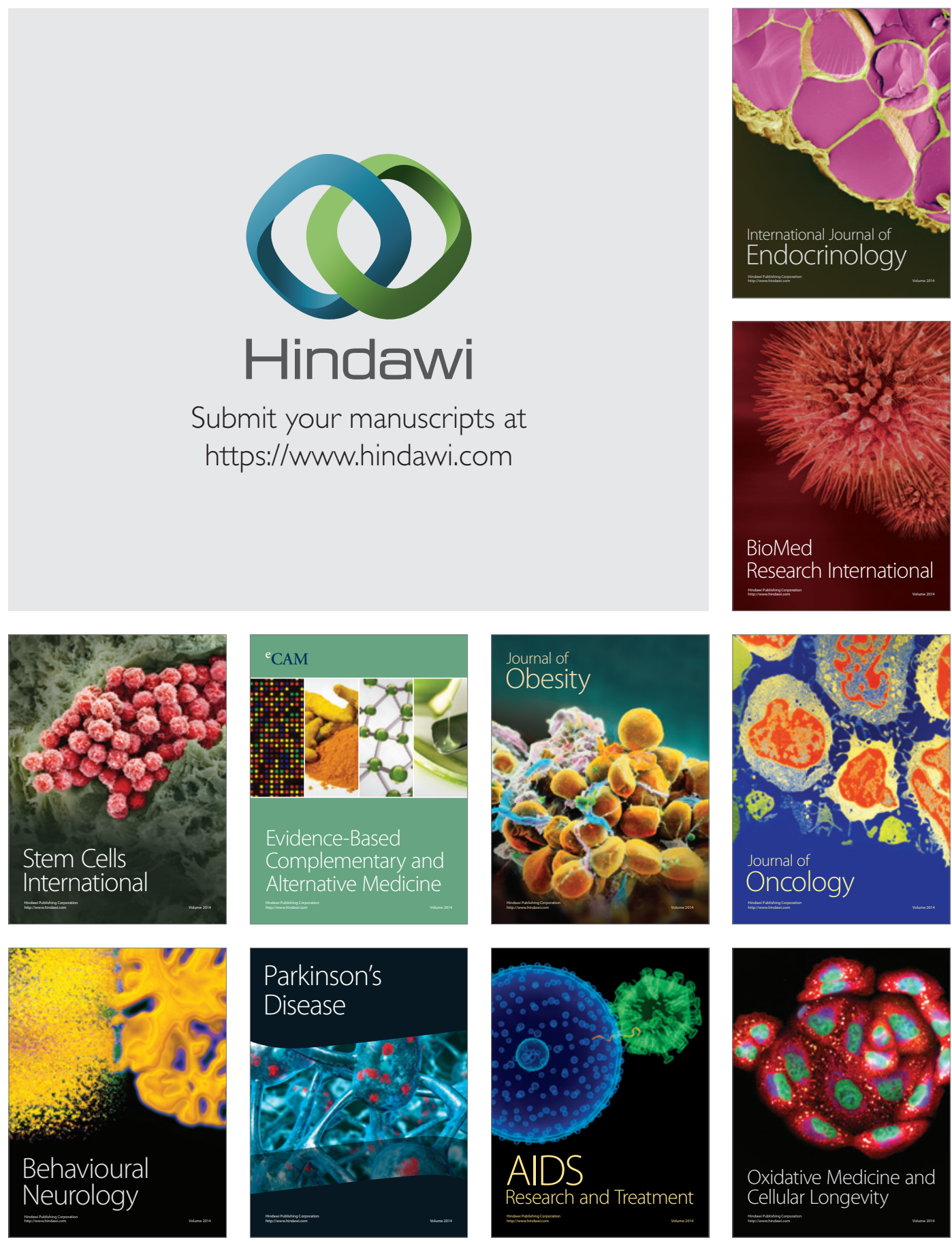